\title{
Measurement of long-pulse relativistic electron beam perpendicular- to-parallel velocity ratio by Cerenkov emission and radiation darkening on a glass plate
}

\author{
J. J. Choi, R. M. Gilgenbach, T. A. Spencer, P. R. Menge, and C. H. Ching \\ Intense Energy Beam Interaction Laboratory, Nuclear Engineering Department, \\ The University of Michigan, Ann Arbor, Michigan 48109-2104
}

(Received 18 January 1991; accepted for publication 29 October 1991)

\begin{abstract}
We report measurements of the ratio of the perpendicular velocity to the parallel velocity, $\alpha$ $=v_{1} / v_{\|}$, of a relativistic electron beam gyrating in a magnetic field by the use of (1)

Cerenkov emission from a glass plate, detected by a gated microchannel plate image intensifier camera, and (2) electron-beam-induced radiation darkening pattern on the same glass plate. The measurements are based on a direct determination of the Larmor radius of an electron beam of known energy. Experiments were performed on a long-pulse electron beam accelerator with $e$-beam diode parameters: $V_{D}=0.6-0.9 \mathrm{MV}$, pulse length $=0.5-1 \mu \mathrm{s}$, $I_{D}=1-10 \mathrm{kA}$. The experimental value of $\alpha$ agrees with simulation results from particle trajectory codes as well as theoretical predictions from Busch's theorem and adiabatic theory.
\end{abstract}

\section{INTRODUCTION}

Relativistic electron beams produced by cold field emission cathodes are important in a number of research areas, such as high-power microwave generation by electron cyclotron resonance masers (CRM), ${ }^{1}$ gas laser pumping, ${ }^{2}$ and fusion plasma heating. ${ }^{3}$ In a CRM, the resonance condition between the $e$ beam and the waveguide mode in a cavity is sensitive to the ratio of the perpendicular velocity to the parallel velocity, $\alpha=v_{1} / v_{\|}$, of the $e$ beam gyrating in an external magnetic field, because the radiated microwave frequency is $\omega=\Omega_{e} / \gamma+k_{\|} v_{\|}$, where $v_{\| l}$ is the axial velocity, $\Omega_{e} / \gamma$ is relativistic cyclotron frequency, and $k_{\|}$is the axial wave number of the beam cyclotron mode. Thus, the beam velocity ratio $\alpha$, ranging from 0.2 to 2 in CRM devices, and its spread are important parameters to accurately measure in such devices.

The transverse electron momentum and its spread have been previously diagnosed by the use of the $\mathrm{x}$-ray radiation pattern. ${ }^{4}$ Cerenkov plates have been utilized elsewhere for emittance measurements. ${ }^{5}$

We describe here a new technique of determining the beam velocity ratio by the use of Cerenkov emission from a glass plate diagnostic with a gated microchannel plate image intensifier camera. The electron beam induced radiation darkening also provides good estimation of the $\alpha$.

\section{THEORY}

When an intense beam of electrons moves through a dielectric material, such as a glass plate, at a speed $(v)$ exceeding that of light in the substance, Cerenkov radiation is emitted in a cone of half angle $\theta$, with $\cos \theta=1 / \beta n$, where $n$ is the index of refraction of the dielectric material, $\beta$ is $v / c$, and $c$ is the light velocity in free space. The beam for a CRM has a certain pitch angle (typically $10^{\circ}-60^{\circ}$ ) when entering the glass convertor, so that the Cerenkov cone is tilted as depicted in Fig. 1(a). As the $e$ beam loses its kinetic energy in the medium, the cone angle becomes smaller. Unlike fluorescence or luminescence, ${ }^{6}$ Cerenkov radiation requires that the beam energy exceed a threshold in order to generate light. The threshold $e$-beam energy is given as $E_{\mathrm{th}}=m_{0} c^{2}\left[\left(1-n^{-2}\right)^{-1 / 2}-1\right]$, where $m_{0}$ is the rest mass. For glass $(n=1.45)$, the cone angle is about $40^{\circ}$ for $\beta=0.9$ and $E_{\mathrm{th}}$ is about $190 \mathrm{keV}$ which is well below the typical electron beam energies obtained from most pulsed power $e$-beam accelerators.

In high current CRMs, the electron beam is produced from an explosive field emission cold cathode in a field immersed diode. The beam is adiabatically compressed as it propagates from the cathode to the interaction region along an increasing externally applied magnetic field, as shown in Fig. 2. During compression, the $e$ beam conserves its canonical angular momentum in an axisymmetric system (Busch's theorem):

$$
P_{\theta}=\left(m \Omega_{e} / 2\right)\left(r_{L}^{2}-r_{0}^{2}\right),
$$

where $\Omega_{e}=e B / m$ is the nonrelativistic cyclotron frequency, $r_{L}$ is the Larmor radius, and $r_{0}$ is the beam guiding center radius. One can relate the Larmor radius of an $e$ beam in the interaction region to the beam radius and the applied magnetic field as

$$
r_{L 2}=\sqrt{r_{2}^{2}+\left(B_{1} / B_{2}\right)\left(r_{L 1}^{2}-r_{1}^{2}\right)}
$$

where $r_{L 1}\left(r_{L 2}\right)$ is the Larmor radius in the diode (in the interaction region), $r_{1}$ is the beam radius at the cathode, $r_{2}$ is the beam guiding center in the interaction region, and $B_{1}$ and $B_{2}$ are the external magnetic fields at the cathode and interaction region, respectively. If we know the injected beam energy $\gamma=\left[1-\left(\beta_{\perp}^{2}+\beta_{\|}^{2}\right)\right]^{-1 / 2}$ and the Larmor radius $r_{L}=\beta_{\perp} \gamma c / \Omega_{e}$, where $\beta_{\perp}=v_{\perp} / c$ and $\beta_{\|}$ $=v_{\|} / c$, we can express the beam velocity ratio as

$$
\alpha=\frac{\beta_{1}}{\beta_{\|}}=\left(\frac{\left(\gamma^{2}-1\right) c^{2}}{\Omega_{e}^{2} r_{L}^{2}}-1\right)^{-1 / 2},
$$

where a monoenergetic electron beam was assumed during the adiabatic compression. 
(a)

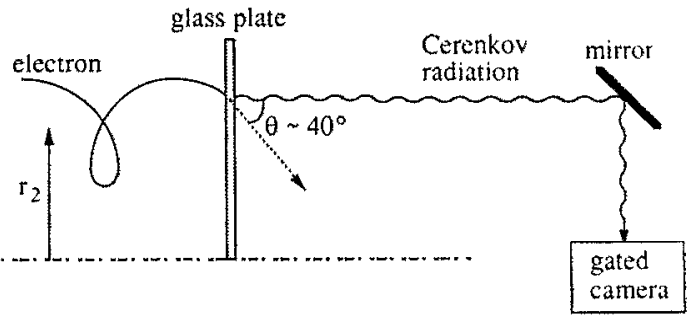

(b)

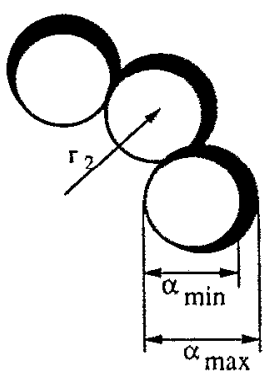

FIG. 1. (a) Schematic of Cerenkov light emission; (b) schematic of electron-beam-induced radiation darkening left on the glass plate.

When the electron beam is adiabatically compressed, the magnetic moment $\mu=m v_{1}^{2} / 2 B$ is invariant. This allows us $^{7}$ to write the beam velocity ratio in the interaction region, $\alpha_{2}$ in terms of $\alpha_{1}$ :

$$
\alpha_{2}=\left(\frac{1+\alpha_{1}^{2}}{\alpha_{1}^{2}} \frac{B_{1}}{B_{2}}-1\right)^{-1 / 2} .
$$

The spread of axial momentum $P_{\|}$and transverse momentum $P_{1}$, is related to the spread of $\alpha$ through

$$
\frac{\left|\Delta P_{\|}\right|}{P_{\|}}=\frac{|\Delta \alpha|}{\alpha} \frac{\alpha^{2}}{1+\alpha^{2}}
$$
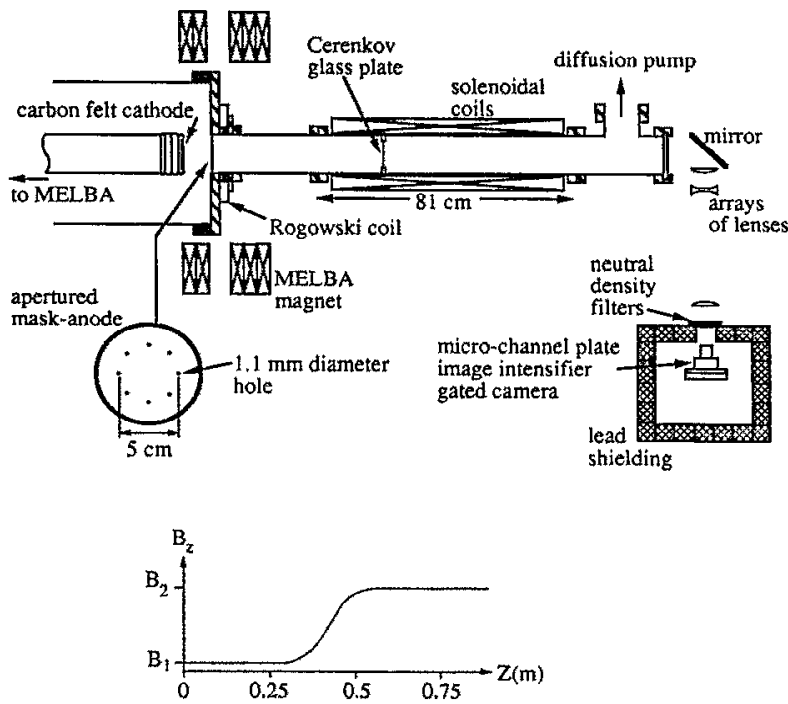

FIG. 2. Upper: experimental configuration for measurements. The Cerenkov glass plate is placed $50 \mathrm{~cm}$ away from the mask anode; lower: magnetic fields pulsed from two capacitor banks. and

$$
\frac{\left|\Delta P_{1}\right|}{P_{1}}=\frac{|\Delta \alpha|}{\alpha} \frac{1}{1+\alpha^{2}}
$$

because $\Delta P_{\perp}=-\Delta P_{\|} / \alpha$. By taking the derivative of $\gamma_{\|}$ $=\left(1-\beta_{\|}^{2}\right)^{-1 / 2}$, one can derive the axial energy spread as

$$
\frac{\left|\Delta \gamma_{\|}\right|}{\gamma_{\|}}=\frac{\left|\Delta P_{\|}\right|}{P_{\|}} \frac{\beta_{\|}^{2}}{1-\beta_{\|}^{2}},
$$

where $\Delta P_{\|}$or $\Delta \alpha$ can be estimated from the spread of the Larmor radius. In deriving the equations of the momentum/energy spreads, a monoenergetic electron beam is assumed, or $d \gamma / \gamma=0$. It should be pointed out that the expression derived for the momentum/energy is only approximate because of the lack of perfectly constant experimental beam energy. However, as will be shown in the section of experimental results, since the beam voltage is very flat, Eqs. (5) and (6) are reasonably appropriate. The diode current does not change rapidly enough that inductive effects on $\gamma$ need to be considered.

As described in the following section, the beam velocity ratio $\alpha$ can be determined from a direct measurement of the Larmor radius and compared with simulation results from an electrostatic particle trajectory $\operatorname{code}^{8}$ and the MAGIC electromagnetic PIC code. ${ }^{9}$

\section{EXPERIMENTAL CONFIGURATION}

A sketch of the experimental configuration is shown in Fig. 2. A pulsed high-power electron beam was produced by the Michigan Electron Long Beam Accelerator (MELBA) ${ }^{10}$ with $e$-beam parameters $V_{D}=0.6-0.9 \mathrm{MV}$, pulse length $=0.5-1 \mu \mathrm{s}, I_{D}=1-10 \mathrm{kA}$. Cathode voltage is measured using a balanced, water/copper-sulphate resistive divider. The planar cathode is made from carbon felt and has a $7.6 \mathrm{~cm}$ diameter with an aluminum shield which covers the edge of the carbon felt cathode. The anodecathode gap is $6.9 \mathrm{~cm}$. The entire surface of the aluminum cathode except the beam carbon felt emitting area is coyered with three coats of glyptal, an electrically insulating enamel. This glyptal coating helps prevent undesired electron field emission from the aluminum. To select a highquality electron beam from a large area solid beam, we have designed an apertured mask anode (Fig. 2), consisting of eight holes (hole diameter $=0.11 \mathrm{~cm}$ ) in a $0.32-\mathrm{cm}$ thick graphite anode plate (POCO graphite, DFP-2). The apertures are equally spaced in a ring of radius $2.52 \mathrm{~cm}$ from the center of the anode plate. The ring radius, equal to $2.52 \mathrm{~cm}$, is optimized for the cavity mode and $\alpha$ required for CRM operation. The apertures, which have a diameter of $0.11 \mathrm{~cm}$ (which is one-third of the plate thickness), permit only the electron beam with $\alpha<0.33$ to pass through the anode. Each hole allows about $1 \mathrm{~A}$ of electron beam to pass through, measured by a Rogowski coil placed in the flange directly after the mask anode. Current density is estimated at $\sim 100 \mathrm{~A} / \mathrm{cm}^{2}$ which is about the same as that reported by Samlin. "The magnetic field coils in the diode and uniform region are independently pulsed from two different capacitor banks, with the profile shown in 
Fig. 2. It should be pointed out that the electron beam with eight beamlets is not axisymmetric and thus the canonical angular momentum in Eq. (1), $P_{\theta}$, is not strictly conserved. Therefore, the comparison between our measurements and the Busch's theorem is only approximate.

The Cerenkov convertor is made of slide cover glass (from Kodak, typically Schott type B-270) with a thickness of $0.13 \mathrm{~cm}$. Patterson et al. ${ }^{12}$ and Downey et al. ${ }^{13}$ have shown that glass is an excellent dielectric material as a Cerenkov convertor. A glass plate is chosen because it has several advantages: (1) the glass has an excellent time response to light emission (fast rise time and fast decay time) and a good correlation of light output to electron current density, ${ }^{11}$ which are important for use with a time-resolved gated camera; (2) electron-beam-induced color change in the glass (radiation darkening) indicates the time-integrated beam pattern; and (3) a glass plate is low cost (slide cover glass) and easy to fashion. To prevent exposure due to the diode plasma light, the side of the glass plate facing the diode was coated with Aerodag, a colloidal carbon spray, until it became opaque. In addition, the carbon coating prevents charge buildup on the glass surface.

The glass plate is located in the uniform field region where the perpendicular velocity of the $e$ beam is maximized by adiabatic compression. The Cerenkov radiation generated is directed by a $90^{\circ}$ turning mirror, focused through an array of lenses, and then recorded on a microchannel plate (MCP) camera. ${ }^{14}$ The MCP camera is a modified Hasselblad single-lens refiex camera with a microchannel plate, proximity focused on a Polaroid film back with an $80 \mathrm{~mm}$ lens. To obtain a time-resolved picture, the light is shuttered by gating the microchannel plate image intensifier by a $40 \mathrm{~ns}$ pulse. The timing of the MCP gate is set to sample the Cerenkov light during the flat-top portion of voltage pulse. Neutral density (ND) light attenuation filters (typically, ND = 3-3.1) are placed in front of the MCP camera to avoid overexposure of the photocathode. A lead bunker shields the MCP camera from $\mathrm{x}$ rays generated by the $e$ beam.

\section{EXPERIMENTAL RESULTS AND DISCUSSION}

Figure 3 represents data from a glass plate exposed to a single shot, $680 \mathrm{kV}, 8 \mathrm{~A}$, eight aperture electron beam for over $0.6 \mu$ s pulse length. The externally pulsed magnetic fields are $0.5 \mathrm{kG}$ in the cathode region and $5 \mathrm{kG}$ in the uniform region. The image of the MCP camera shows nearly all beamlets clearly [Fig. 3(a)], indicating a fairly uniform current distribution. As shown in Fig. 3(b), electron-beam-induced radiation darkening was observed in the area subject to the electron beam trajectories. Since the radiation darkening was observed with well-defined color change (brown), we did not have to use a densitometer. The time-integrated beam pattern is a direct measure of the beam Larmor radius in an external magnetic field. Because the MELBA beam voltage is almost flat over $0.6 \mu$ s pulse length, this integrated radiation darkening is a good diagnostic to examine the beam behavior throughout the whole voltage pulse. The reproducibility of such a clear radiation darkening image was excellent as long as the beam voltage (a)

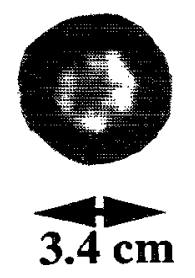

(b)

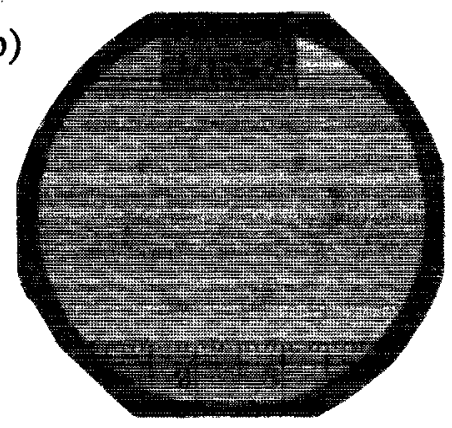

(c)

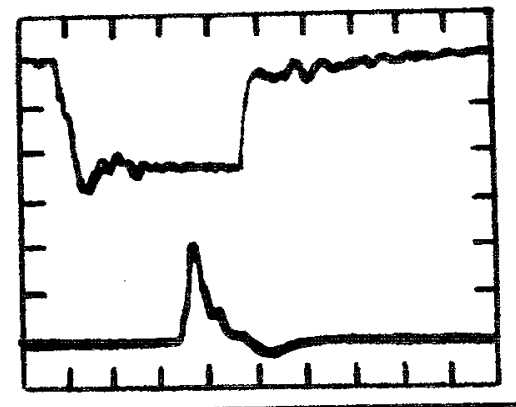

(e)

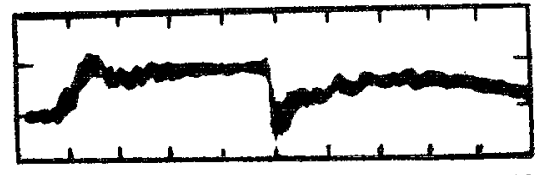

(f)

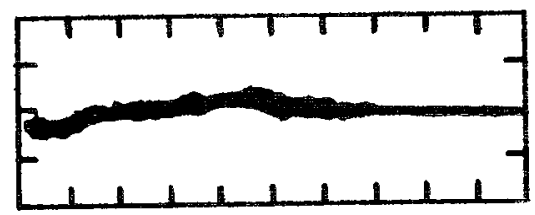

(g)

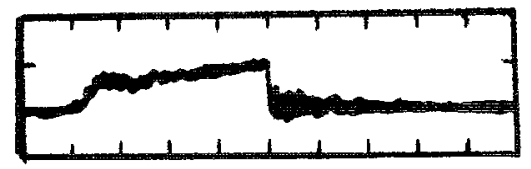

FIG. 3. (a) MCP camera photograph of Cerenkov light, (b) radiation darkening on Cerenkov glass plate, (c) MELBA beam voltage (310 kV/ div), (d) gate of MCP camera, (e) cathode stalk current ( $4.5 \mathrm{kA} / \mathrm{div})$, (f) apertured beam current $(40 \mathrm{~A} / \mathrm{div})$, and $(\mathrm{g})$ anode current $(3.5$ $\mathrm{kA} / \mathrm{div}$ ). The magnetic fields are $B_{1}=0.5 \mathrm{kG}$ and $B_{2}=5 \mathrm{kG}$.

was flat over the whole pulse length. The optical density of the pattern was uniform for all eight beamlets. It was observed that the radiation darkening left on the glass faded at room temperature after several months. 
Based on the direct visual measurement of the Larmor radius of $0.35 \mathrm{~cm}$ from the center of the radiation darkened rings on the glass plate, we determined the beam velocity ratio, $\alpha$ of 0.56 where the beam kinetic energy was $680 \mathrm{kV}$ $(\gamma=2.33)$ during the flat top of the voltage pulse. To compare the data with the theoretical prediction, we measured the beam guiding center radii, $r_{1}=2.52 \mathrm{~cm}$ and $r_{2}$ $=0.9 \mathrm{~cm}$. The beam velocity ratio directly after the apertured mask anode, $\alpha_{1}=0.16$, was obtained from the computer simulation by MAGIC electromagnetic PIC code. ${ }^{9}$ The emission threshold electric field was assumed to be 20 $\mathrm{kV} / \mathrm{cm}$ on the fabric emitting surface and $700 \mathrm{kV} / \mathrm{cm}$ on the glyptal-coated cathode stalk. A uniform clectron emission on the 7.6-cm-diam cathode was assumed. Using Eq. (2) we obtained $r_{L 2}=0.4 \mathrm{~cm}$, which is consistent with the measured Larmor radius. In addition, the adiabatic theory, as shown in Eq. (4), predicted $\alpha_{2}=0.58$, which is in good agreement with the measured $\alpha_{2}=0.56$. One can interpret the thickness of the radiation darkened rings [Fig. 3(b)] as a spread of the guiding center location because of the "finite" size of the holes and the $e$-beam perpendicular energy spread. The measured thickness of $\sim 1 \mathrm{~mm}$ from the radiation darkening allows an estimate of the upper bound in the spread of a Larmor radius of $\pm 0.5 \mathrm{~mm}$, corresponding to a beam velocity ratio spread of \pm 0.05 . The calculated momentum and energy spread from Eqs. (5) and (6) are $\Delta P_{\|} / P_{\|} \leqslant 4 \%, \Delta P_{\perp} / P_{\perp} \leqslant 14 \%$, and $\Delta \gamma_{\|} / \gamma_{\|} \leqslant 7 \%$, respectively, indicating that a high-quality $e$ beam with low velocity spread and low axial energy spread is extracted from the apertured mask anode. Note that for a beam with $\alpha$ $<1$, most of the beam velocity spread comes from the transverse momentum spread, whereas for a beam with $\alpha>1$, the beam velocity spread is mostly from the axial momentum spread. This is the intrinsic characteristic of an adiabatic magnetic compression of the electron beam, which is seen in Eq. (5).

The Stanford EGUN code ${ }^{8}$ is used for comparison with our $\alpha$ measurement. Figure 4(a) shows the $r-z$ trajectory predicted by the code for a single $680 \mathrm{kV}$ beam from the apertured mask anode which propagates into a grounded cylindrical drift tube in the downstream uniform magnetic field, $5 \mathrm{kG}$, with the diode magnetic field, $B_{1}$ $=0.5 \mathrm{kG}$. Uniform cathode emission was turned on in the code in the region of the fabric cathode. Figure 4(b) shows the predicted $\alpha$ for different diode magnetic fields when $B_{2}$ is fixed at $5 \mathrm{kG}$ and $B_{1}$ is adjusted from 0.3 to $0.9 \mathrm{kG}$. When $B_{1}=0.5 \mathrm{kG}$, the code predicts the beam velocity ratio, $\alpha_{2}=0.66$, which is in reasonable agreement with the measured $\alpha_{2}=0.56 \pm 0.05$. In addition, the beam guiding center radius after compression is found to be $0.9 \mathrm{~cm}$, which agrees with the measured guiding center, $r_{2}$ $=0.82 \pm 0.25 \mathrm{~cm}$. Although the EGUN code assumes an axisymmetric system, the comparison between the measurements and code results shows reasonably good agreement. The values of $\alpha$ obtained in the present experiments are consistent with values obtained from frequency measurements of backward wave cyclotron maser microwave emission.

Increasing the magnification factor of the optical lens

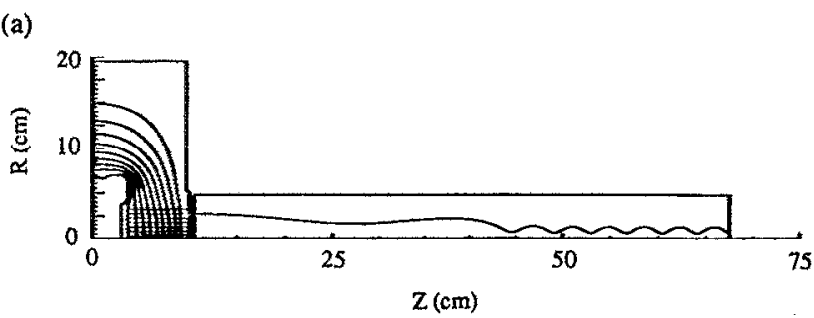

(b)

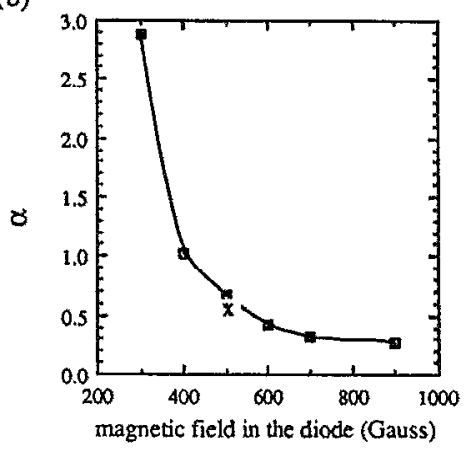

FIG. 4. (a) Electron trajectory plot from Hermannsfeldt code $(680 \mathrm{kV}$, $B_{1}=0.5 \mathrm{kG}, B_{2}=5 \mathrm{kG}$ ); (b) plot of computed beam velocity ratio (D) vs magnetic field in the cathode region. The experimentally measured $\alpha$ (denoted by $\times$ ) is plotted for $B_{1}=0.5 \mathrm{kG}$.

array made it possible to see a large image of a Larmor orbit (see Fig. 5). In this shot, we applied the magnetic field of $B_{1}=0.7 \mathrm{kG}$ and $B_{2}=5 \mathrm{kG}$. The beam guiding center radius agreed well with the one directly measured from the radiation darkening although each Larmor orbit from the MCP photograph shows a brightly filled circle, rather than an annular ring. We noticed that the radiation darkening produced a much better image of the Larmor orbit than the MCP photograph. This is because the Cerenkov light emission broadens with a large angular spread as the electrons randomly scatter in the glass convertor. A glass much thinner than an electron range may produce a better time resolved image. Another difficulty in the time-resolved measurements is that the electron-beam-induced radiation darkening reduces the temporal Cerenkov emission by absorption in the glass during the pulse. However, the Cerenkov emission was intense enough so that neutraldensity attenuators were still required. It should be noted that there were some other difficulties in gathering data from the MCP camera, such as image focusing on the thin glass convertor, image resolution of the MCP photocathode and adjusting light intensity by the use of neutral density light attenuation filters. Thus we mainly relied on the

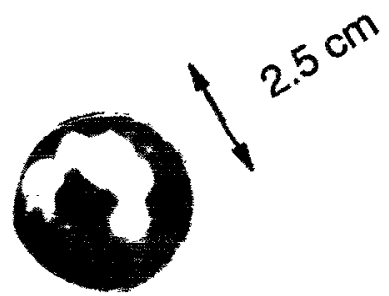

FIG. 5. Magnified Larmor orbits from MCP camera photograph. The magnetic fields are $B_{1}=0.7 \mathrm{kG}$ and $B_{2}=5 \mathrm{kG}$. 
(a)
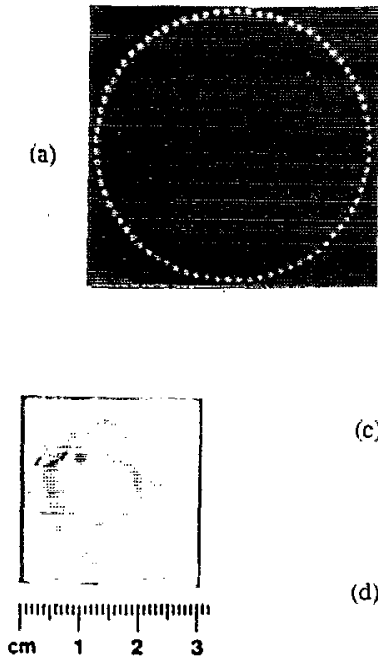

(c)

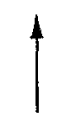

$5.04 \mathrm{~cm}$
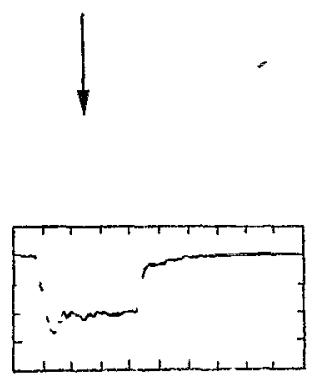

(d)

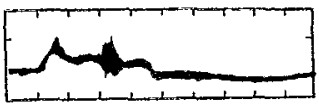

FIG. 6. (a) Apertured mask anode with 80 holes, (b) radiation darkening pattern, (c) beam voltage ( $310 \mathrm{kV} / \mathrm{div})$, and (d) apertured beam current ( $40 \mathrm{~A} / \mathrm{div})$.

radiation darkening pattern in the glass plate, especially when examining the spread of the beam energy and momentum.

Based on the results obtained from the 8-hole apertured mask anode, we fabricated an 80-hole apertured mask anode with the same diameter and hole position from the anode center [see Fig. 6(a)]. A single shot with a 620 $\mathrm{kV}$ and 30-50 A electron beam with pulsed magnetic fields $B_{1}=0.5 \mathrm{kG}$ and $B_{2}=4.7 \mathrm{kG}$ produced a radiation darkening pattern, as is shown in Fig. 6(b). The observed beam guiding center and Larmor radius are $0.85 \mathrm{~cm}$ and 0.35 $\mathrm{cm}$, respectively. Thus the beam velocity ratio is determined to be 0.56 . This second apertured mask anode with

known $\alpha$ has been used to perform high-power CRM microwave experiments. ${ }^{15}$

\section{ACKNOWLEDGMENTS}

This research was supported in part by the Air Force Office of Scientific Research, Air Force Phillips Laboratory, and Los Alamos National Laboratory. We gratefully acknowledge useful discussions with Dr. R. Bosch.

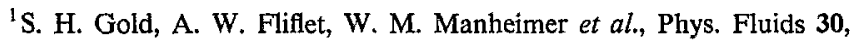
2226 (1987); G. Bekefi, A. DiRienzo, C. Leibovitch, and B. G. Danly, Appl. Phys. Lett. 54, 1302 (1989).

${ }^{2}$ L. A. Rosocha and K. B. Riepe, Fusion Tech. 11, 576 (1987).

${ }^{3}$ R. M. Gilgenbach, M. E. Read, K. E. Hackett et al., Phys. Rev. Lett. 44, 647 (1980); R. M. Gilgenbach, M. E. Read, K. E. Hackett et al., Nucl. Fusion 21, 319 (1981).

${ }^{4}$ S. Talmadge, T. C. Marshall, and S. P. Schlesinger, Phys. Fluids 20, 974 (1977).

${ }^{5}$ G. Bekefi, F. Hartemann, and D. A. Kirkpatrick, J. Appl. Phys. 62, 1564 (1987).

${ }^{6}$ V. I. Krementsov, P. S. Strelkov, and A. G. Shkvarunets, Sov. Phys. Tech. Phys. 25, 1447 (1980).

${ }^{7}$ C. Kyler and K. R. Chu, Naval Research Lab. Memorandum Report 4401, 1981.

${ }^{8}$ W. B. Herrmannsfeldt, SLAC-226, November, 1979.

${ }^{9}$ B. Goplen, L. Ludeking, J. McDonald, G. Warren, and R. Worl, MAGIC User's Manual (Mission Research Corporation, Newington, 1989).

${ }^{10}$ R. M. Gilgenbach, L. D. Horton, R. F. Lucey, S. Bidwell, M. Cuneo, J. Miller, and L. Smutek, Proc. 5th IEEE Pulsed Power Conference, June 10-12, 1985, p. 126.

${ }^{11}$ G. E. Samlin, J. Appl. Phys. 62, 2169 (1987).

${ }^{12}$ E. L. Patterson, R. A. Hamil, and G. E. Samlin, J. Appl. Phys. 54, 515 (1983).

${ }^{13}$ S. W. Downey, L. A. Builta, R. L. Carlson, S. J. Czuchlewski, and D. C. Moir, J. Appl. Phys. 60, 3460 (1986).

${ }^{14}$ S. Hauver, R. E. Pechacek, J. R. Greig, D. P. Murphy, and M. Raleigh, Naval Research Lab. Memorandum Report 5702, 1985.

${ }^{15}$ J. J. Choi, R. M. Gilgenbach, and T. A. Spencer, Proc. of 15 th International Conference on Infrared \& MM Waves, SPIE 1514, 143 (1990). 